\title{
Prevalence, risk factors, and impact of lung Cancer on outcomes of idiopathic pulmonary fibrosis: a study from the Middle East
}

\author{
Sherif Mohamed ${ }^{1 *}$ (D) Hassan Bayoumi ${ }^{1}$, Nashwa Abd El-Aziz² ${ }^{2}$ Ehab Mousa ${ }^{3}$ and Yasser Gamal ${ }^{4}$
}

\begin{abstract}
Background: No studies have addressed the impact of lung cancer (LC) on prognosis of patients with idiopathic pulmonary fibrosis (IPF) in Upper Egypt. We aimed to evaluate the prevalence and risk factors for LC among IPF patients and its impact on their outcomes and survival in Upper Egypt.

Methods: A total of 246 patients with IPF who had complete clinical and follow up data were reviewed. They were categorized into 2 groups: 34 patients with biopsy-proven LC and IPF (LC-IPF) and 212 patients with IPF only (IPF). Survival and clinical characteristics of the two groups were compared.

Results: Prevalence of LC was 13.8\%. Pack/years was the most significant predictor for LC development in IPF (Odds ratio; 3.225, Cl 1.257-1.669, $p=0.001$ ). Survival in patients with LC-IPF was significantly worse than in patients with IPF without LC; median survival, 35 months vs 55 months; $p=0.000$. LC accompanying IPF was one of the most significant independent predictors of survival in IPF patients (Hazard ratio 5.431, Cl 2.186-13.492, $p=0.000$ ). Mortality in LC-IPF patients was mainly due to LC progression in $36 \%$ and LC therapy-related complications in $22 \%$.

Conclusions: Prevalence of LC in IPF patients was 13.8\%. Lung cancer has significant impacts on patients with IPF in Upper Egypt, in terms of clinical outcomes and survival. Smoking is the most significant independent predictor of LC development in IPF patients. A poorer survival was observed for patients with IPF developing LC, mainly due to LC progression, and to complications of its therapies. Further prospective, multicenter and larger studies are warranted.
\end{abstract}

Keywords: Prevalence, Impact, Lung cancer, Idiopathic pulmonary fibrosis, Survival, Upper Egypt

\section{Background}

Idiopathic pulmonary fibrosis (IPF) is the most common of the idiopathic interstitial pneumonias and carries the worst prognosis [1]. Despite that several clinicopathologic and radiographic variables have been shown to correlate with survival in patients with IPF [2], the development of lung cancer (LC) among those patients further worsens their prognosis $[3,4]$.

Previous studies have addressed the clinical risk factors associated with LC development in IPF patients [3, 5-7]. A higher incidence of LC has been described in older male

\footnotetext{
* Correspondence: saawm220@gmail.com

${ }^{1}$ Department of Chest Diseases and Tuberculosis, Faculty of Medicine, Assiut University, Assiut 71516, Egypt

Full list of author information is available at the end of the article
}

smokers and in patients with combined pulmonary fibrosis and emphysema (CPFE) $[3,5,7,8]$. However, tumor location in patients with both LC and IPF (LC-IPF) differs among studies [3-7]. Furthermore, there is no consensus regarding the most prevalent histologic type of LC-IPF [6-10].

Previous studies had compared LC profiles in patients with and without IPF [3-10]. However, these studies included only populations from Asian [3, 6-9] and Western $[4,5,10]$ countries. There is considerable lack of such studies in the Egyptian and/or Middle East populations. Ethnical differences could exist [11-13]. Therefore, in the current study, we aimed to evaluate the prevalence and risk factors for LC among patients with IPF and to address possible impact for LC on clinical outcomes and long-term survival of IPF patients in Upper Egypt. 


\section{Methods}

\section{Study design and population}

Assiut University Hospital (AUH) and South Egypt Cancer Institute (SECI) are two large tertiary hospitals serving a large number of populations at Upper Egypt.

A systematic search of the patient database at the Chest Department of AUH revealed 267 patients who fulfilled the international guidelines on the diagnosis and management of IPF [1], during the period of July 1, 2012, to July 31, 2017. Among these 267 patients, we selected 246 patients diagnosed with IPF that were then followed up regularly at our Department according to a prospective protocol for follow up of IPF patients, including clinical management, annual high resolution computed tomography (HRCT) scanning, and regular pulmonary function testing (PFT). The remaining 21 patients were excluded due to incomplete follow up data.

These 246 cases were categorized in two groups: (1) the group of 34 patients who had (at the time of diagnosis of IPF) or developed LC (biopsy-proven) during the follow up period, named the LC-IPF group. Once diagnosed with LC, the patients were referred to the Medical Oncology Department, SECI, for further oncologic management, and (2) the group of 212 patients with IPF only (IPF group). The 2 groups were compared for clinical features, outcomes, and survival analysis. The later was carried out in all cases from the time of IPF diagnosis.

\section{Data collection and diagnostic criteria}

The medical records of all IPF patients were reviewed to obtain data regarding age, smoking history, the method used to diagnose IPF, time of IPF diagnosis, PFT, HRCT findings, comorbidities, follow up duration, and outcome. Pack/years value was calculated by multiplying the number of packs of cigarettes smoked per day by the number of years the person has smoked [14]. The findings of HRCT scans were classified as reticular, honeycomb, ground-glass opacities, and nodular patterns. An acute exacerbation of IPF (AEIPF) was defined as acute respiratory worsening for which a cause could not be identified and meeting all criteria for as proposed by the international agreement [15], and their updates [16]. Patients diagnosed as combined pulmonary fibrosis and emphysema (CPFE) were not enrolled in the study.

For the LC-IPF group, the time of diagnosis and location of LC including the IPF-associated area were analyzed. All cases of LC were biopsy-proven. Lung cancers were classified according to the World Health Organization classification. Staging of LC has been established by the TNM system current at the time of diagnosis. Side effects of treatments were assessed using the National Cancer Institute Common Terminology Criteria for Adverse Events (CTCAE, version 4.0) [17]. Evaluation of tumor response to chemotherapy was assessed according to the Response
Evaluation Criteria in Solid Tumors (RECIST) criteria; version 1.1 [18]. Mortality related to either surgical intervention and/or oncologic treatment was defined as death occurring within 30 days of treatment. Date of death was verified using hospital records, local death registry review, and review of subsequent patient visits.

The study was approved by the Ethical Committee of AUH and SECI.

\section{Statistical analysis}

Patient demographics were compared using the unpaired t-test for continuous variables and the Pearson's $\chi^{2}$ test for categorical variables. Logistic regression analysis was performed to assess the risk of LC in IPF patients. Survival was estimated using Kaplan-Meier curves. The survival rates of patients in the IPF only group and the IPF with lung cancer (LC-IPF) group were compared using a log-rank test. Cox's proportional hazards regression analysis was used to identify significant variables that affect the survival of IPF patients and to estimate hazard ratios (HR) and 95\% confidence intervals (CI) for predictors of survival. All tests were two-tailed, and $p<0.05$ were considered statistically significant. SPSS software Version 22.0 (SPSS Inc., Chicago, IL, USA) was used for all statistical analyses.

\section{Results}

\section{Patient characteristics}

The prevalence of LC in IPF patients was $13.8 \%$. Table 1 summarizes the clinical findings in IPF patients grouped according to the presence or absence of LC. The mean age at diagnosis of IPF was $52.4 \pm 8.2$ years, and $69.5 \%$ of IPF patients were males. When the 2 groups were compared, there were significant differences in age, smoking status, pack/years, forced vital capacity (FVC), forced expiratory volume in $1 \mathrm{~s}\left(\mathrm{FEV}_{1}\right)$, diffusing capacity of the lung for carbon monoxide $\left(\mathrm{D}_{\mathrm{LCO}}\right)$, and AEIPF.

\section{HRCT and clinicopathologic findings in LC-IPF patients} Among the 34 patients with LC-IPF, 12 (35.3\%) were diagnosed as having primary pulmonary LC at the same time of IPF diagnosis. The other 22 (64.7\%) patients developed LC $19.4 \pm 16.8$ months (median, 32 months; range, 3.2-78.4 months) after diagnosis of IPF during the follow up period. The cumulative incidence of $\mathrm{LC}$ at 1 and 3 years was 37.2 and $62.5 \%$, respectively.

Most of the cancerous lesions were located in peripheral areas of the lung (73.5\%). Two-thirds (67.6\%) of the lesions were in the lower lobes. Also, two-thirds of the cancerous lesions were located in a fibrotic background (IPF-associated areas) in the same patients. Squamous cell carcinoma (44\%) and adenocarcinoma (41\%) were the most common histological findings in LC-IPF patients. 
Table 1 Demographics of patients with idiopathic pulmonary fibrosis in the 2 study groups

\begin{tabular}{|c|c|c|c|c|}
\hline Characteristic & All IPF & IPF and LC & IPF only & $P$ \\
\hline Subjects No. (\%) & $246(100)$ & $34(13.8)$ & $212(86.2)$ & 0.000 \\
\hline Age, $\pm S D, y$ & $52.4 \pm 8.2$ & $56.6 \pm 10.0$ & $47.7 \pm 10.2$ & 0.002 \\
\hline Gender No. (\%) & & & & 0.107 \\
\hline Male & $171(69.5)$ & 28(82.3) & $143(67.4)$ & \\
\hline Female & $75(30.5)$ & $6(17.7)$ & 69 (32.6) & \\
\hline Smoking status & & & & 0.033 \\
\hline Current & $180(73.2)$ & $22(64.6)$ & $158(74.4)$ & \\
\hline Past & $31(12.6)$ & $4(11.8)$ & $27(12.8)$ & \\
\hline Never & $35(14.2)$ & $8(23.6)$ & $27(12.8)$ & \\
\hline Pack/years & $33.4 \pm 16.7$ & $37.2 \pm 21.7$ & $30.7 \pm 13.1$ & 0.016 \\
\hline FVC (\% pred) & $80.8 \pm 2.9$ & $85.9 \pm 2.7$ & $77.1 \pm 3.8$ & 0.000 \\
\hline $\mathrm{FEV}_{1}(\%$ pred $)$ & $82.3 \pm 5.0$ & $87.0 \pm 4.8$ & $76.9 \pm 5.9$ & 0.010 \\
\hline TLC (\% pred) & $69.2 \pm 10.1$ & $70.1 \pm 12.8$ & $67.8 \pm 9.3$ & 0.206 \\
\hline $\mathrm{D}_{\mathrm{LCO}}(\%$ pred $)$ & $60.2 \pm 8.3$ & $57.1 \pm 10.4$ & $62.6 \pm 9.5$ & 0.000 \\
\hline Diagnostic method & & & & 0.021 \\
\hline Clinical & $218(88.6)$ & $26(76.5)$ & 192 (90.6) & \\
\hline Surgical & $28(11.4)$ & $8(23.5)$ & $20(9.4)$ & \\
\hline AEIPF & 87 (35.3) & $18(53)$ & $69(32.5)$ & 0.032 \\
\hline
\end{tabular}

Data are presented as mean $\pm S D$, percentage or median (min-max) unless otherwise indicated. \% pred, percentage of the predicted value; FVC; forced vital capacity, FEV ; forced expiratory volume in $1 \mathrm{~s}$, TLC; total lung capacity,

$\mathrm{D}_{\mathrm{LCO}}$; diffusing capacity of the lung for carbon monoxide, AEIPF; acute

exacerbations of idiopathic pulmonary fibrosis

Eight (23.3\%) patients had early stage I lung cancer. Five (15\%), 9(26.4\%), and 12(35.3\%) patients had stage II, III, and IV, respectively. Table 2 shows the radiological and clinicopathological findings in LC-IPF patients.

\section{Risk factor analysis of lung cancer}

To assess possible risk factors for the development of LC in IPF patients, variables including age, gender, smoking, pack/years, and $\mathrm{D}_{\mathrm{LCO}}$ were analyzed. Multivariate regression after adjustment for significant factors revealed that pack/years and male gender were significant independent predictors of LC in IPF patients (Table 3).

\section{Therapies for LC and their complications}

Out of the 34 patients with LC-IPF, 4 underwent surgery, while 5,2,13, and 10 received radiotherapy, radiochemotherapy, chemotherapy, and best supportive care, respectively. The decisions for different therapeutic modalities were undertaken by a multidisciplinary team, taking into consideration the underlying IPF. A total of 17/24 (71\%) patients experienced therapy-related complications. Surgery for early LCs consisted of four lobectomies. Three patients (75\%) had complications, mainly pneumonia. Estimated 30 -day mortality was $25 \%$. Complications were reported in $3 / 5$ (60\%), $1 / 2(50 \%)$, and $10 / 13(77 \%)$ of patients who
Table 2 HRCT findings, histopathology and stages of LC in patients with LC-IPF $(n=34)$

\begin{tabular}{|c|c|}
\hline Characteristic & No. (\%) \\
\hline \multicolumn{2}{|l|}{ Time of LC diagnosis } \\
\hline At the time of IPF diagnosis & $12(35.3)$ \\
\hline After IPF diagnosis & $22(64.7)$ \\
\hline \multicolumn{2}{|l|}{ Location } \\
\hline Central $^{a}$ & $9(26.5)$ \\
\hline Peripheral & $25(73.5)$ \\
\hline \multicolumn{2}{|l|}{ Lobes of the lung } \\
\hline Upper lobes & $10(29.5)$ \\
\hline Middle lobe & $1(2.9)$ \\
\hline Lower lobes & $23(67.6)$ \\
\hline \multicolumn{2}{|l|}{ HRCT background } \\
\hline Fibrotic background ${ }^{\mathrm{b}}$ & $23(67.6)$ \\
\hline Nonfibrotic background & $11(32.4)$ \\
\hline \multicolumn{2}{|l|}{ Histopathology } \\
\hline Squamous cell carcinoma & $15(44)$ \\
\hline Adenocarcinoma & $14(41)$ \\
\hline Adenosquamous carcinoma & $2(6)$ \\
\hline large cell carcinoma & $2(6)$ \\
\hline Undifferentiated carcinoma & $1(3)$ \\
\hline \multicolumn{2}{|l|}{ Stage } \\
\hline । & $8(23.3)$ \\
\hline$\|$ & $5(15.0)$ \\
\hline III & $9(26.4)$ \\
\hline IV & $12(35.3)$ \\
\hline
\end{tabular}

LC-IPF combined lung cancer and idiopathic pulmonary fibrosis, HRCT highresolution computed tomography

${ }^{a}$ Central; the tumor was considered central when located in an area $3 \mathrm{~cm}$ far from the pleura

${ }^{\mathrm{b}}$ Fibrotic background; includes reticular distortion, ground-glass opacity, and honeycombing

underwent radiotherapy, radio-chemotherapy, and chemotherapy, respectively. Those who received radio- or radio-chemotherapy were among the 11 patients with non-fibrotic lung pathology. Among 13 patients treated with chemotherapy only, 5 (38.5\%) patients died with a

Table 3 Multivariate analysis of risk factors for predicting the development of lung cancer in idiopathic pulmonary fibrosis

\begin{tabular}{llll}
\hline Variables & OR & $95 \% \mathrm{Cl}$ & $P$ - \\
\hline Gender, male & 1.089 & $0.014-0.579$ & 0.011 \\
Age, & 0.677 & $0.214-9.757$ & 0.707 \\
Smoking & 0.411 & $0.378-12.869$ & 0.279 \\
Pack/year & 3.225 & $1.257-1.669$ & 0.001 \\
DLCO & 0.849 & $1.016-1.138$ & 0.062 \\
\hline
\end{tabular}

Cl confidence interval, $O R$ odds ratio, $D_{L C O}$ diffusing capacity of the lung for carbon monoxide 
median survival of 7 months (range, 3-22 months). Table 4 details these results. Patients with LC-IPF did not receive antifibrotic therapy (pirfenidone/nintedanib) for their IPF.

\section{Survival analysis}

Among the 212 patients with IPF without LC, 93(43.8\%) died, median survival was 55.0 months, while in the group of patients with LC-IPF, 28(82.3\%) patients died, median survival was 35.0 months. Survival time was significantly different between the 2 groups ( $p=0.000$, log-rank test). One- and 3-year survivals among the two groups were $90 \%$ and $52 \%$ in the LC-IPF group and 98 and $83 \%$ in the IPF group, respectively. Survival curves of patients with IPF with and without LC are displayed in Fig. 1.

Lung cancer accompanying IPF was one of the most significant independent predictors of survival in IPF patients (HR 5.431, CI 2.186-13.492, $p=0.000$ ). The other significant predictor was smoking (HR 2.114, CI $1.370-5.118, p=0.004)$. Table 5 summarizes the HR of each variable in the total sample of patients with IPF.

In the LC-IPF group, six (22\%) patients died of lethal complications related to LC therapies. Four (14\%) died of AEIPF. Ten (36\%) patients died of LC progression, and

Table 4 Therapy-related complications and acute exacerbations of IPF in LC-IPF patients $(n=34)$

\begin{tabular}{|c|c|c|c|}
\hline Complications & $\%$ & $\begin{array}{l}\text { AEIPF, } \\
\text { No. (\%) }\end{array}$ & $\begin{array}{l}\text { Mortality of AEIPF, } \\
\text { No. (\%) }\end{array}$ \\
\hline $\begin{array}{l}\text { Surgery-related } \\
\text { complications }(n=3 / 4)\end{array}$ & $75 \%$ & $2 / 4(50)$ & $1 / 2(50)$ \\
\hline Respiratory failure & $25 \%$ & & \\
\hline Pneumonia & $50 \%$ & & \\
\hline Empyema & $25 \%$ & & \\
\hline Myocardial infarction & $25 \%$ & & \\
\hline $\begin{array}{l}\text { Radiotherapy-related } \\
\text { complications }(n=3 / 5)\end{array}$ & $60 \%$ & $2 / 5(40)$ & $0 / 2(0)$ \\
\hline Radiation pneumonitis & $33 \%$ & & \\
\hline Pulmonary infections & $33 \%$ & & \\
\hline Respiratory failure & $17 \%$ & & \\
\hline Myocardial infarction & $17 \%$ & & \\
\hline $\begin{array}{l}\text { Radio-chemotherapy-related } \\
\text { complications }(n=1 / 2)\end{array}$ & $50 \%$ & $0 / 2(0)$ & $0 / 1(0)$ \\
\hline Radiation pneumonitis & $50 \%$ & & \\
\hline $\begin{array}{l}\text { Chemotherapy-related } \\
\text { complications }(n=10 / 13)\end{array}$ & $77 \%$ & $8 / 13(61.5)$ & $5 / 8(62.5)$ \\
\hline Pancytopenia & $30 \%$ & & \\
\hline Pneumonia & $40 \%$ & & \\
\hline Respiratory failure & $20 \%$ & & \\
\hline Cardiovascular failure & $25 \%$ & & \\
\hline Total & $71 \%$ & $12 / 24(50)$ & $6 / 12(50)$ \\
\hline
\end{tabular}

LC lung cancer, IPF idiopathic pulmonary fibrosis, AEIPF acute exacerbation of idiopathic pulmonary fibrosis

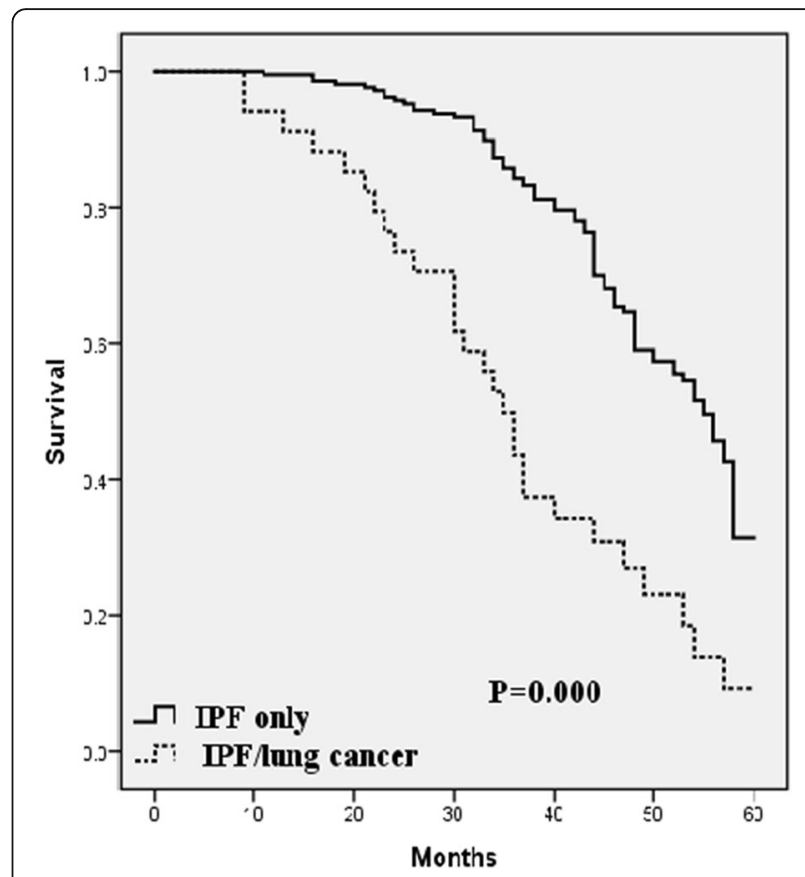

Fig. 1 Comparison of survival between idiopathic pulmonary fibrosis with lung cancer (LC-IPF) patients and idiopathic pulmonary fibrosis (IPF) only patients; Kaplan-Meier survival curve

eight (28\%) patients died of respiratory failure related to IPF.

\section{Progression of IPF}

Median follow up duration was 32 months (range, 1.060.0 months) in the LC-IPF group and 42.6 months (range, 6.0-98.2 months) in the IPF group. During the follow up period, 24 patients among 34 with LC (70.6\%) and 122 among 212 without LC (57.5\%) experienced IPF progression. Median time-to-disease progression was 24 months in the LC-IPF group and 27 months in the IPF group. There was not a significant difference in progression-free survival between the two groups; $p=$ 0.087. After adjusting for age, gender, smoking status, \% predicted $\mathrm{D}_{\mathrm{LCO}}$, and \% predicted $\mathrm{FVC}$, there was no difference (HR 1.359; 95\% CI, 0.774-2.338; $p=0.131$ ).

\section{AE of IPF}

A total of 87 patients (35.3\%) experienced AEIPF during the study period, with a significant difference between the 2 groups; $18 / 34$ (53\%) were in the LC-IPF group, and $69 / 212(32.5 \%)$ were in the IPF group, $p=0.032$, respectively. (Table 1) Mortality for AE was 55.6\% (10 of 18 ) and $71 \%$ (49 of 69) in LC-IPF and IPF-only, respectively. Notably, in patients with LC-IPF, AEs were triggered by therapy for LC in $50 \%$ of cases (12/24); two surgery (of four patients, 50\%), two radiotherapies (of five patients, 40\%), and 8 chemotherapies (of 13 patients, 61.5\%). Mortality due to treatment-related AEs was 
Table 5 Cox regression analysis for predictors of survival in patients with idiopathic pulmonary fibrosis

\begin{tabular}{llll}
\hline Variables & Hazard Ratio & $95 \% \mathrm{Cl}$ & $P$ \\
\hline Gender & 0.615 & $0.318-1.192$ & 0.150 \\
Age & 1.015 & $0.991-1.040$ & 0.224 \\
Smoking & 2.114 & $1.370-5.118$ & 0.004 \\
Pack/year & 0.998 & $0.968-1.029$ & 0.880 \\
FVC (\% pred) & 1.018 & $0.964-1.001$ & 0.057 \\
FEV ${ }_{1} \%$ pred) & 1.008 & $0.957-1.062$ & 0.766 \\
TLC (\% pred) & 0.702 & $0.956-1.014$ & 0.298 \\
DLCO $_{\text {(\% pred) }}$ & 1.148 & $0.319-4.966$ & 0.067 \\
Lung cancer & 5.431 & $2.186-13.492$ & 0.000 \\
\hline
\end{tabular}

$\mathrm{Cl}$ confidence interval, FVC forced vital capacity, \% pred; percentage of predicted value, $F E V_{1}$ forced expiratory volume in $1 \mathrm{~s}, T L C$ total lung capacity, $D_{L C O}$ diffusing capacity of the lung for carbon monoxide

reported in 50\% (5/10) of cases. Table 4 shows AEIPF related to LC therapies.

\section{Discussion}

To the best of our knowledge, this is the first study that addresses the impact of LC on IPF patients in the Middle East and Egypt. Results showed that LC has significant impacts on clinical outcomes and survival of patients with IPF in Upper Egypt. Demographic features of patients with IPF and/or LC could be different in our locality. Mohamed and Ibrahim [11], in their study of 568 patients with IPF in Upper Egypt, concluded that IPF has a different age and sex distribution in Upper Egypt, compared to international data. It was observed that $43 \%$ of patients with IPF developed their disease before the age of 50 , with a mean age of $48.6 \pm 12.9$ years at the time of diagnosis [12]. In a recent study for primary LC in Upper Egypt, it was observed that the incidence of adenocarcinoma surpassed that of squamous cell carcinoma, and it was common that the patients presented with their illness at later stages [13].

Our data revealed that the prevalence of LC in IPF patients is $13.8 \%$ in all $(8.9 \%$ in LC onset during the IPF follow up). The cumulative incidence of LC among IPF patients at 1 and 3 years was 37.2 and $62.5 \%$, respectively. Our results are consistent with the worldwide reported prevalence range of 4.8 to $48 \%[3-5,7]$. Moreover, these data represent the third report; after those of Ozawa et al. [19] and Tomassetti et al [4], documenting that cumulative incidence of $\mathrm{LC}$ increases remarkably overtime after the initial diagnosis of IPF. Furthermore, these data confirm the crucial importance of a prospective protocol for follow up of IPF patients with annual HRCT scanning.

It has been observed that $\mathrm{LC}$ was more common among IPF patients who are older, males, and smokers. These findings are consistent with those of previous reports enrolling different populations $[3-7,9,10]$. For these reasons, we think that those particular populations with IPF should receive intensified follow up protocols for the development of LC. Despite that tumor location in LC-IPF patients was different among studies [3-7], results of the current study are in agreement with the majority of other studies of LC in IPF [3-10]. Most of the cancerous lesions were peripherally located, in the lower lobes, and in IPF-associated lesions. These findings add more support to the theory that the inflammatory process is associated with bronchiolar metaplasia in the pathogenesis of LC [20]. Again, we believe that those populations deserve more attention during their follow up protocols. It is necessary to raise awareness of LC risk among patients with IPF.

Similarly, there was no consensus regarding the most prevalent histologic type of LC-IPF [3-10]. Squamous cell carcinoma was the most common histological type encountered by some authors $[4,5,10]$, while adenocarcinoma was encountered by others [21, 22]. Squamous cell carcinoma and adenocarcinoma were found in 44 and $41 \%$ of our LC-IPF cohort, respectively. In a recent systemic review, Wang et al. concluded that adenocarcinoma and squamous cell carcinoma are the most common types in IPF-associated LC patients [23].

Considering the clinical, radiologic, and pathologic findings of our cohort together may support the current hypothesis that considers IPF as a disease of premature aging with several links to $\operatorname{LC}[4,5,8,24]$. However, the pathogenesis of combined IPF and LC is not so simple. Recently, it has been proposed that perturbed signaling pathways, epigenetic and genetic changes, oxidative stress, and fibroblast growth factor receptor (FGFR) signaling pathways are all thought to be involved in the pathogenesis of IPF-associated LC [23].

Previous studies have highlighted the clinical risk factors associated with LC development in IPF patients [3-7, 25]. It was consistently shown that elderly male IPF patients with a history of smoking are more likely to develop LC. In this context, we observed that pack/years and male gender were significant independent predictors of LC in IPF patients. A study from England suggested that the incidence of LC is significantly increased in IPF patients compared to the general population, and that smoking is an independent predictor of LC development [26].

Survival analysis of our cohort showed interesting results. Survival time was significantly different between LC-IPF and IPF only $(p=0.000)$. LC accompanying IPF was one of the most significant independent predictors of survival in IPF patients (HR 5.431, CI 2.186-13.492, $p=0.000$ ).

Notably, the difference in mortality seen in our study was not attributable to worsening of pulmonary fibrosis, but mainly to both LC progression and complications of LC therapies. Similar to the findings observed by Tomassetti et al. [4], this is the second report that confirms such significant findings. 
Our results are in strong agreement with previous reports [3-7]. Lee and coworkers [3] concluded that LC was the most predictor for mortality among IPF patients (HR 2.441, CI 1.373-4.339; $p=0.002$ ), while Tomassetti et al [4], observed a statistically significant difference in survival of patients with LC-IPF compared with IPF only, with an adjusted HR of 7 (95\% CI, 3.81-12.90; $p=0.001$ ).

The majority of deaths among LC-IPF patients seen in the current study was not due to worsening of pulmonary fibrosis, but to both LC progression and complications of LC treatment; in 36 and $22 \%$ of patients, respectively. Moreover, it was observed that $50 \%$ of AEs in patients with $\mathrm{LC}$ were triggered by therapies for LC. These risks make the decisions about LC management in IPF, despite undertaken by a multidisciplinary team, still challenging to the clinician.

Furthermore, two-thirds of patients with LC-IPF (23/ $34,67.6 \%)$ had advanced cancer and received either chemotherapy (with a high rate of complications) or best supportive care. Despite improvements in our understanding of pathogenetic mechanisms for the development of LC among IPF patients [23], the current treatment strategies for combined IPF and LC, including medications and surgery are still controversial, complex and thorny $[4,23,27]$.

Several studies had addressed the impact of surgical resection of LC in patients with IPF. Early postoperative mortality ranged from 0 to $18.2 \%$ and postoperative morbidity ranged from 7.1 to $40.7 \%$ [27, 28]. Notably, we reported surgery-related complications in $75 \%$ of operated patients, mainly pneumonia. Kreuter and colleagues reported an incidence of $67 \%$ for surgery-related complications and a 30-day mortality of $25 \%$ among the operated patients with LC-IPF [10].

This high rate of surgery-related complications, again highlights the importance of annual HRCT for patients with IPF, for early detection of LC which might give the patient better surgical therapeutic options, hence better outcomes.

There are only sparse data concerning radiotherapy in LC with IPF [10]. Despite those patients who received radio-, or radio-chemotherapy had non-fibrotic ground in HRCT still, the reported rates of radiation pneumonitis and pulmonary infections were high, but with no impact on mortality. Local tumor ablation might be an attractive option in some of those severely pulmonary compromised patients, while it has been used safely in patients with severe emphysema [29]. Disappointingly, it seems that chemotherapy is not a safe therapeutic option for patients with LC-IPF. Chemotherapy-related toxicities were reported in $77 \%$ of our cohort, mainly pneumonia, and pancytopenia. Previous studies reported chemotherapy-related toxicities in 63\% [10] and 50\% [4] of patients with LC-IPF.
Acute exacerbations have significant impact on IPF patients, particularly those with LC-IPF, with considerable morbidity and mortality. In the literature, the incidence of treatment-related AEs in patients with LC-IPF ranged between 12.5 and $30 \%$ and mortality of treatment-related $\mathrm{AE}$ ranged between 9 and 16\% [30, 31]. However, our results revealed a higher incidence of treatment-related AEs $(50 \%)$ and their related mortality (50\%). Careful monitoring of patients for these AEs, and if possible, their prediction [32], are warranted.

Despite the current study is the first one in Egypt and the Middle East that describes the impact of LC on IPF in a unique group of populations, yet it is to be considered that the inherent disadvantage of being a retrospective study, together with its single-center design, and the relatively limited number of patients are important limitations. The impact of LC on IPF and the optimal management of LC in patients with IPF needs to be addressed in larger prospective multicenter studies. Finally, our data from Upper Egypt are generally similar to those previously reported from Western and Asian populations.

\section{Conclusions}

Lung cancer has significant impact on patients with IPF in Upper Egypt, in terms of clinical outcomes and survival. Smoking and male gender were significant independent predictors of lung cancer development in IPF patients. A poorer survival rate was observed in patients with IPF developing LC, mainly due to LC progression, and to complications of its treatments. Chemotherapy and surgery had a high incidence of fatal complications. Careful monitoring of the patients for these complications is needed. Further prospective, multicenter and larger studies are warranted.

\section{Abbreviations \\ AEIPF: Acute exacerbation of idiopathic pulmonary fibrosis; CPFE: Combined pulmonary fibrosis and emphysema; CTCAE: Common Terminology Criteria for Adverse Events; D Lco: Diffusing capacity of the lung for carbon monoxide; FEV : Forced expiratory volume in $1 \mathrm{~s}$; FGFR: Fibroblast growth factor receptor; FVC: Forced vital capacity; HR: Hazard ratio; HRCT: High resolution computed tomography; IPF: Idiopathic pulmonary fibrosis; LC: Lung cancer; LC-IPF: Combined lung cancer and idiopathic pulmonary fibrosis; OR: Odds ratio; PFT: Pulmonary function tests; RECIST: Response evaluation criteria in solid tumors; TLC: Total lung capacity; TNM: Tumor, node, metastasis; WHO: World Health Organization}

\section{Funding}

This research did not receive any specific grant from funding agencies in the public, commercial, or not-for-profit sectors.

\section{Availability of data and materials}

The datasets used and/or analyzed during the current study are available from the corresponding author on reasonable request.

\section{Authors' contributions}

All authors have provided approval of the manuscript to be published. SM, HB, and NA contributed to the draft of the submitted article, revised it critically for important content, and made substantial contributions to conception and design, as well as data acquisition, analysis, and interpretation; SM and EM 
contributed to the draft of the submitted article and made substantial contributions to acquisition, analysis, and interpretation of radiologic data; SM and YG contributed to the draft of the submitted article and made substantial contributions to acquisition, analysis, and interpretation of pathology data. All authors read and approved the final manuscript.

\section{Ethics approval and consent to participate}

The study was approved by the Ethical Committee of AUH and SECI. Written informed consent was provided by each subject before participation. All procedures conformed to the standards set by the Declaration of Helsinki in its latest revision.

\section{Consent for publication}

Not applicable.

\section{Competing interests}

The authors declare that they have no competing interests.

\section{Publisher's Note}

Springer Nature remains neutral with regard to jurisdictional claims in published maps and institutional affiliations.

\section{Author details}

'Department of Chest Diseases and Tuberculosis, Faculty of Medicine, Assiut University, Assiut 71516, Egypt. ${ }^{2}$ Department of Medical Oncology, South Egypt Cancer Institute, Assiut University, Assiut, Egypt. 'Department of Radiology, Faculty of Medicine, Assiut University, Assiut, Egypt. ${ }^{4}$ Department of Pathology, Faculty of Medicine, Assiut University, Assiut 71516, Egypt.

\section{Received: 3 July 2018 Accepted: 17 August 2018}

Published online: 03 October 2018

\section{References}

1. Raghu G, Collard HR, Egan JJ, Martinez FJ, Behr J, Brown KK, et al. ATS/ ERS/ JRS/ALAT Committee on idiopathic pulmonary fibrosis. An official ATS/ ERS/ JRS/ALAT statement: idiopathic pulmonary fibrosis: evidence-based guidelines for diagnosis and management. Am J Respir Crit Care Med. 2011; 183:788-824.

2. Ley B, Collard HR, King TE Jr. Clinical course and prediction of survival in idiopathic pulmonary fibrosis. Am J Respir Crit Care Med. 2011;183:431-40.

3. Lee KJ, Chung MP, Kim YW, Lee JH, Kim KS, Ryu JS, et al. Prevalence, risk factors and survival of lung cancer in the idiopathic pulmonary fibrosis. Thorac Cancer. 2012;3:150-5.

4. Tomassetti S, Gurioli C, Ryu JH, Decker PA, Ravaglia C, Tantalocco P, et al. The impact of lung Cancer on survival of idiopathic pulmonary fibrosis. Chest. 2015;147:157-64.

5. Aubry MC, Myers JL, Douglas WW, Tazelaar HD, Washington Stephens TL, Hartman TE, et al. Primary pulmonary carcinoma in patients with idiopathic pulmonary fibrosis. Mayo Clin Proc. 2002;77:763-70.

6. Nagai A, Chiyotani A, Nakadate T, Konno K. Lung cancer in patients with idiopathic pulmonary fibrosis. Tohoku J Exp Med. 1992;167:231-7.

7. Park J, Kim DS, Shim TS, Lim CM, Koh Y, Lee SD, et al. Lung cancer in patients with idiopathic pulmonary fibrosis. Eur Respir J. 2001;17:1216-9.

8. Usui $K$, Tanai C, Tanaka Y, Noda H, Ishihara T. The prevalence of pulmonary fibrosis combined with emphysema in patients with lung cancer. Respirology. 2011;16:326-31.

9. Lee HJ, Im JG, Ahn JM, Yeon KM. Lung Cancer in patients with idiopathic pulmonary fibrosis: CT findings. J Comput Assist Tomogr. 1996:20:979-82.

10. Kreuter M, Ehlers-Tenenbaum S, Schaaf M, Oltmanns U, Palmowski K, Hoffmann $\mathrm{H}$, et al. Treatment and outcome of lung cancer in idiopathic interstitial pneumonias. Sarcoidosis Vasc Diffuse Lung Dis. 2015;31:266-74.

11. Mohamed AR, Ibrahim AK. Idiopathic pulmonary fibrosis (IPF) in upper Egypt, a single center study. Egypt J Chest Dis Tuberc. 2015;64:915-9.

12. Rifaat N, Hasan AA. Scope on idiopathic pulmonary fibrosis in upper Egypt. Egypt J Broncho. 2015;9:154-9.

13. Omar A, Abo Elfadl A, Ahmed Y, Hamed R, Zaky AH. Primary lung cancer in Assiut University Hospitals: pattern of presentation within four years (January 2011: December 2014). Egypt J Chest Dis Tuberc. 2017;66:675-80.

14. National Cancer Institute definition of pack year. https://www.cancer.gov/ publications/dictionaries/cancer-terms/def/pack-year.
15. Collard HR, Moore BB, Flaherty KR, Brown KK, Kaner RJ, King TE, et al. Idiopathic pulmonary fibrosis clinical research network investigators. Acute exacerbations of idiopathic pulmonary fibrosis. Am J Respir Crit Care Med. 2007:176:636-43.

16. Collard HR, Ryerson CJ, Corte TJ, Jenkins G, Kondoh Y, Lederer DJ, et al. Acute exacerbation of idiopathic pulmonary fibrosis. An international working group report. Am J Respir Crit Care Med. 2016;194:265-75.

17. National Cancer Institute. Common Terminology Criteria for Adverse Events (CTCAE), version 4.0; 2010. https://www.eortc.be/services/doc/ctc/CTCAE_4. 03_2010-06-14_QuickReference_5x7.pdf.

18. Eisenhauer EA, Therasse P, Bogaerts J, Schwartz LH, Sargent D, Ford R, et al. New response evaluation criteria in solid tumors: revised RECIST guideline (version 1.1). Eur J Cancer. 2009;45:228-47.

19. Ozawa Y, Suda T, Naito T, Enomoto N, Hashimoto D, Fujisawa T, et al. Cumulative incidence of and predictive factors for lung cancer in IPF. Respirology. 2009;14:723-8. https://doi.org/10.1111/j.1440-1843. 2009.01547.x.

20. Hironaka M, Fukayama M. Pulmonary fibrosis and lung carcinoma: a comparative study of metaplastic epithelia in honeycombed areas of usual interstitial pneumonia with or without lung carcinoma. Pathol Int. 1999:49:1060-6.

21. Kawai T, Yakumaru K, Suzuki M, Kageyama K. Diffuse interstitial pulmonary fibrosis and lung cancer. Acta Pathol Jpn. 1987;37:11-9.

22. Ma Y, Seneviratne CK, Koss M. Idiopathic pulmonary fibrosis and malignancy. Curr Opin Pulm Med. 2001;7:278-82.

23. Wang Y, Liu Y, Zhang Q, Gao R, Wang K. IPF and lung cancer: homologous but different endings, the progress in the correlation research. Int J Clin Exp Med. 2017:10:4319-29.

24. Chilosi M, Carloni A, Rossi A, Poletti V. Premature lung aging and cellular senescence in the pathogenesis of idiopathic pulmonary fibrosis and COPD/emphysema. Transl Res. 2013;162:156-73.

25. Lee T, Park JY, Lee HY, Cho YJ, Yoon H, Lee JH, et al. Lung cancer in patients with idiopathic pulmonary fibrosis: clinical characteristics and impact on survival. Respir Med. 2014;108:1549-55.

26. Jeune IL, Gribbin J, West J, Smith C, Cullinan P, Hubbard R. The incidence of cancer in patients with idiopathic pulmonary fibrosis and sarcoidosis in the UK. Respir Med. 2007;101:2534-40.

27. Watanabe A, Higami T, Ohori S, oyanagi T, Nakashima S, Mawatari T. Is lung cancer resection indicated in patients with idiopathic pulmonary fibrosis? J Thorac Cardiovasc Surg. 2008;136:1357-63.

28. Saito Y, Kawai Y, Takahashi N, Ikeya T, Murai K, Kawabata Y, et al. Survival after surgery for pathologic stage IA non-small cell lung cancer associated with idiopathic pulmonary fibrosis. Ann Thorac Surg. 2011;92:1812-7.

29. Schneider T, Puderbach M, Kunz J, Bischof A, Giesel FL, Dienemann H, et al. Simultaneous computed tomography-guided biopsy and radiofrequency ablation of solitary pulmonary malignancy in high-risk patients. Respiration. 2012;84:501-8.

30. Watanabe N, Taniguchi H, Kondoh Y, Kimura T, Kataoka K, Nishiyama O, et al. Efficacy of chemotherapy for advanced non-small cell lung cancer with idiopathic pulmonary fibrosis. Respiration. 2013;85:326-31.

31. Kenmotsu H, Naito T, Kimura M, Ono A, Shukuya T, Nakamura Y, et al. The risk of cytotoxic chemotherapy-related exacerbation of interstitial lung disease with lung cancer. J Thorac Oncol. 2011;6:1242-6.

32. Kobayashi H, Omori S, Nakashima K, Wakuda K, Ono A, Kenmotsu H, et al. Modified GAP index for prediction of acute exacerbation of idiopathic pulmonary fibrosis in non-small cell lung cancer. Respirology. 2017;22:1379-85.

Ready to submit your research? Choose BMC and benefit from:

- fast, convenient online submission

- thorough peer review by experienced researchers in your field

- rapid publication on acceptance

- support for research data, including large and complex data types

- gold Open Access which fosters wider collaboration and increased citations

- maximum visibility for your research: over $100 \mathrm{M}$ website views per year

At $\mathrm{BMC}$, research is always in progress.

Learn more biomedcentral.com/submission 